\title{
A IMPORTÂNCIA DA ATENÇÃO PRIMÁRIA NA REDUÇÃO DE GASTOS EM DOENÇAS CARDIOVASCULARES NO SISTEMA ÚNICO DE SAÚDE
}

\section{THE IMPORTANCE OF PRIMARY CARE TO REDUCE EXPENSES IN THE UNIFIED HEALTH SYSTEM}

Ariana leda Lima Ferreira da Silva, Diego Henrique Mota Cordeiro de Souza, Leonardo Ito Yui, Nathália Marcussi Oliveira

Universidade do Oeste Paulista - UNOESTE, Faculdade de Medicina, Presidente Prudente, SP. E-mail: nathalia marcussi@hotmail.com.

\section{RESUMO}

A atenção básica deve ser preferencialmente a porta de entrada para o Sistema Único de Saúde, sendo que a Estratégia da Saúde da Família (ESF) configura-se como a principal modalidade de atuação da Atenção Básica (Tanaka, 2011). A ação da Atenção Básica vai ter papel importante na prevenção de algumas DCNT muito prevalentes na população, como o diabetes mellitus e a hipertensão arterial sistêmica. O cuidado clínico das doenças cardiovasculares é dispendioso e prolongado. Esses custos diretos desviam os escassos recursos familiares e sociais para os cuidados médicos. Trata-se de um estudo transversal retrospectivo com análise de dados secundários disponíveis no site DATA SUS com fonte de dados do Ministério da Saúde e SIM. O objetivo deste estudo foi analisar a importância da atenção básica na redução de gastos com tratamentos de doenças cardiovasculares, no Sistema Único de Saúde, através de prevenção e promoção à saúde.

Palavras-chave: Atenção Primária à Saúde, Controle de Custos, Sistema Único de Saúde, Prevenção Primária, Promoção da Saúde. 


\section{INTRODUÇÃO}

As Doenças Crônicas Não Transmissíveis (DCNT) - principalmente doenças cardiovasculares, câncer e doenças respiratórias crônicas - são as principais causas de morte, causando aproximadamente 38 milhões de mortes por ano em todo o mundo (ORGANIZAÇÃO MUNDIAL DE SAÚDE, 2014). O impacto socioeconômico das doenças crônicas está aumentando e é considerado um problema para a saúde pública mundial. Além das mortes prematuras, as DCNT são responsáveis pela incapacidade para o trabalho, pela redução dos rendimentos familiares e pela redução da produtividade (SIQUEIRA, FILHO, LAND, 2017).

Para atingir a meta de redução da mortalidade prematura por DCNT as intervenções mais importantes devem estar focadas na redução da prevalência dos fatores de risco modificáveis. O Plano de Enfrentamento das DCNT do Ministério da Saúde (2011) apresenta diversas ações que visam à promoção da saúde, à prevenção e ao controle dessas doenças. Uma das formas de interação direta quando se fala em prevenção, é por meio de ações de atenção básica à saúde, realizadas pelos núcleos municipais de Estratégia de Saúde da Família - ESF através de equipes multidisciplinares. Essas ações são apontadas pelo Ministério da Saúde como principal estratégia de organização da assistência primária (Giacomin, 2011).

A Política Nacional de Atenção Básica (Brasil, 2006, p. 1) descreve que a atenção básica se caracteriza por um conjunto de ações de saúde, no âmbito individual e coletivo, que abrangem a promoção e a proteção da saúde, a prevenção de agravos, o diagnóstico, o tratamento, a reabilitação e a manutenção da saúde. A atenção básica deve ser preferencialmente a porta de entrada para o Sistema Único de Saúde, sendo que a Estratégia da Saúde da Família (ESF) configura-se como a principal modalidade de atuação da Atenção Básica (Tanaka, 2011).

A ação da Atenção Básica vai ter papel importante na prevenção de algumas DCNT muito prevalentes na população, como o diabetes mellitus e a hipertensão arterial sistêmica. A hipertensão é importante fator de risco, sendo a causa mais frequente das demais doenças do aparelho circulatório. Além disso, a hipertensão está, ainda, associada às demais doenças e condições crônicas, tais como doença renal crônica, diabetes, entre outras (MINISTÉRIO DA SAÚDE, 2011).

A incidência de diabetes cresceu nos últimos anos e já está entre as dez maiores causas de morte em todo o mundo, além de ser uma importante causa de morte por doença cardiovascular. O aumento da taxa de mortalidade devido ao diabetes foi impulsionado pelo crescimento da população idosa, a epidemia de obesidade e estilos de vida não saudáveis (MALTA et al, 2017).

A Estratégia de Saúde da Família representa uma inovação mundial em termos de política universal de Atenção Primária à Saúde. Para que a Atenção Primária à Saúde maximize sua contribuição para a prevenção e controle de doenças crônicas, é imprescindível uma forte atuação nos territórios e entre as populações saudáveis ou de não-doentes (MEDINA et al, 2014). A prevenção e promoção da saúde visa o indivíduo e o coletivo, através da busca de qualidade de vida, autonomia e estímulo ao autocuidado. Percebe-se que a elas se expressam fundamentalmente nas unidades básicas através da educação em saúde. (JANINI, BESSLER, VARGAS, 2015).

O cuidado clínico das doenças cardiovasculares é dispendioso e prolongado. Esses custos diretos desviam os escassos recursos familiares e sociais para os cuidados médicos. A doença cardiovascular afeta os indivíduos em seus períodos médios de vida médios, interrompendo o futuro das famílias dependentes deles e prejudicando o desenvolvimento das nações (ORGANIZAÇÃO MUNDIAL DE SAÚDE, 2014).

O objetivo deste estudo foi analisar a importância da atenção básica na redução de gastos com tratamentos de doenças cardiovasculares, no Sistema Único de Saúde, através de prevenção e promoção à saúde. 


\section{METODOLOGIA}

Trata-se de um estudo transversal retrospectivo com análise de dados secundários disponíveis no site DATA SUS com fonte de dados do Ministério da Saúde e SIM.

Primeiro foram analisadas as principais causas de mortalidade no Brasil classificadas pelo CID-10. Em seguida analisou-se os dados sobre gastos em tratamentos de DCV de acordo com os procedimentos hospitalares do SUS no Brasil. Considerando os custos calculados em moeda brasileira (reais) para o ano de 2016.

Os custos hospitalares de procedimentos incluídos foram: Tratamento De Acidente Vascular Cerebral - AVC (Isquêmico Ou Hemorrágico Agudo; Tratamento Do Acidente Vascular Cerebral Isquêmico Agudo Com Uso De Trombolítico; Tratamento De Infarto Agudo Do Miocárdio; Tratamento De Síndrome Coronariana Aguda; Tratamento De Diabetes Mellitus.

Quanto à organização e ao processamento dos dados, os mesmos foram realizados por meio de análise descritiva simples. Os dados foram apresentados por meio de figuras e tabelas e confrontados com literatura nacional e internacional.

\section{RESULTADOS}

A principal causa de mortalidade entre 2010 e 2015, no Brasil, foram as doenças do aparelho circulatório. Neste período foi registrado um total de 7.190.298 de mortes, as doenças do aparelho circulatório representam $28 \%$ do total, enquanto as neoplasias, segundo maior causa de morte representa $16 \%$.

Os valores encontrados para os custos dos tratamentos de diabetes mellitus foram:

\begin{tabular}{|l|l|}
\cline { 2 - 2 } \multicolumn{1}{c|}{} & TRATAMENTO DE DIABETES MELLITUS \\
\hline 2016 & $57.960 .078,13$ \\
\hline 2015 & $61.567 .489,91$ \\
\hline 2014 & $62.827 .700,40$ \\
\hline 2013 & $63.140 .096,35$ \\
\hline 2012 & $63.226 .346,39$ \\
\hline 2011 & $65.966 .690,33$ \\
\hline
\end{tabular}

Já os custos com doenças cardiovasculares representam valores maiores, os custos para tratamento de infarto agudo do miocárdio aumentaram em 4\% de 2015 a 2016, enquanto para o tratamento de síndrome coronariana aguda reduziu em $2 \%$ nesse mesmo período.

\begin{tabular}{|l|l|l|}
\cline { 2 - 3 } \multicolumn{1}{c|}{} & $\begin{array}{l}\text { TRATAMENTO DE INFARTO AGUDO DO } \\
\text { MIOCÁRDIO }\end{array}$ & $\begin{array}{l}\text { TRATAMENTO DE SINDROME } \\
\text { CORONARIANA AGUDA }\end{array}$ \\
\hline 2016 & $134.871 .935,53$ & $80.149 .077,78$ \\
\hline 2015 & $128.723 .964,23$ & $82.094 .816,54$ \\
\hline 2014 & $119.637 .421,22$ & $83.640 .059,63$ \\
\hline 2013 & $107.712 .386,23$ & $75.307 .073,83$ \\
\hline 2012 & $104.921 .328,81$ & $75.224 .213,02$ \\
\hline 2011 & $97.323 .922,15$ & $65.586 .337,38$ \\
\hline
\end{tabular}


O tratamento do acidente vascular cerebral isquêmico agudo com uso de trombolítico representa a maior proporção de aumento nos valores, subindo 14\% de 2015 para 2016.

\begin{tabular}{|l|l|l|}
\cline { 2 - 3 } \multicolumn{1}{c|}{} & $\begin{array}{l}\text { TRATAMENTO DE ACIDENTE VASCULAR } \\
\text { CEREBRAL - AVC (ISQUEMICO OU } \\
\text { HEMORRÁGICO AGUDO) }\end{array}$ & $\begin{array}{l}\text { TRATAMENTO DO ACIDENTE VASCULAR } \\
\text { CEREBRAL ISQUÊMICO AGUDO COM USO } \\
\text { DE TROMBOLÍTICO }\end{array}$ \\
\hline 2016 & $248.858 .732,58$ & $4.108 .758,76$ \\
\hline 2015 & $238.743 .480,08$ & $3.583 .412,06$ \\
\hline 2014 & $232.334 .709,79$ & $1.788 .787,34$ \\
\hline 2013 & $221.777 .297,31$ & $959.344,36$ \\
\hline 2012 & $210.631 .115,60$ & $4.669,65$ \\
\hline 2011 & $197.929 .125,33$ & NÃO TEM VALOR \\
\hline
\end{tabular}

\section{DISCUSSÃO}

As doenças do coração e dos vasos constituem a primeira causa de morte no Brasil, considerando-se todas as faixas etárias e ambos os sexos, representando $28 \%$ do total de óbitos nos últimos seis anos.

O diabetes mellitus constitui o grupo das doenças crônicas responsáveis pelas principais causas de morte no mundo e é considerado um dos problemas de saúde de maior magnitude (SHAW JE et al., 2010).

O número de diabéticos está aumentando e a grande prevalência de DM na população faz com que suas complicações sejam também frequentes, gerando grande demanda aos serviços de saúde (MALTA et al., 2015). Mesmo com o aumento da prevalência dessa doença, os gastos em tratamentos para diabetes no Brasil reduziu.

A hipertensão arterial sistêmica também faz parte do grupo das doenças crônicas e constitui importante fator de risco para complicações cardíacas e cerebrovasculares, sendo considerada um problema de saúde pública em âmbito mundial. Por ser uma doença crônica, o seu controle requer acompanhamento e tratamento por toda a vida, envolvendo as medidas farmacológicas e não farmacológicas. (RADOVANOVIC, 2014).

A prevalência da HAS aumenta com a idade, multiplicando o risco de danos cardiovasculares, contribuindo para o aumento da morbimortalidade e os custos sociais com invalidez e absenteísmo ao trabalho (MATAVELLI et al., 2012).

A diabetes e hipertensão são condições comumente associados. Essas duas doenças são fatores de risco para desenvolvimento de doenças cardiovasculares, que são a principal causa de morte no Brasil. Sendo essas doenças multifatoriais, é preciso intervir primeiro nos fatores de risco com a prevenção primária e então, caso já haja o desenvolvimento das doenças, é necessário fazer uma prevenção secundária para evitar as complicações.

A atenção básica é a principal responsável pela prevenção e promoção à saúde, devendo atuar principalmente nas populações mais carentes, já que são as mais vulneráveis aos fatores de riscos (RIBEIRO et al., 2016). A orientação, como instrumento da educação em saúde, é um elemento essencial para que as pessoas possam realizar o autocuidado, para terem uma vida saudável e produtiva (SALCl et al., 2017).

A prevenção vai atuar na redução da morbimortalidade por doenças crônicas. Isso tem impacto direto nos custos para a saúde pública. Como evidenciado nos resultados, os valores para tratamento das doenças cardiovasculares são significativamente maiores que o gasto no tratamento de diabetes. Aqui a prevenção secundária tem maior papel, mas a prevenção primária ainda vai agir para controlar a doença, evitando maiores complicações.

A identificação, o tratamento e o controle da hipertensão arterial devem ser considerados determinantes principais para a redução da mortalidade. Especificamente no Brasil, há outro fator 
de risco importante na gênese da doença cerebrovascular: as doenças cardíacas, com forte potencial para embolias (LOTUFO et al., 2017).

A prevenção das DCNTs e de seus fatores de risco é essencial para se reduzir o crescimento dessas doenças e suas consequências, além de causar mortes, elas geram efeitos econômicos adversos para as famílias e as comunidades, assim como para o sistema de saúde, pois este ainda está baseado no modelo do cuidado a eventos agudos (SILOCCHI, JUNGES, 2017).

As DCNT constituem o maior problema de saúde no Brasil, as estimativas indicaram que as perdas de produtividade do trabalho e diminuição da renda familiar resultante de apenas três doenças combinadas (diabetes, doenças cardíacas e acidentes vasculares cerebrais) contribuíram com uma perda de US\$ 4,18 Bilhões para a economia entre 2006 e 2015. Os custos diretos correspondem às despesas envolvidas em assistência médica, medicamentos, hospitalizações, exames, procedimentos, fisioterapia e reabilitação. Os custos indiretos estão ligados a perdas na produção e renda, produtividade, empregos e absenteísmo (MALTA et al., 2017).

\section{CONCLUSÃO}

As DCNT representam uma importante preocupação para o Brasil. A mortalidade de algumas DCNT (as doenças cardiovasculares e as respiratórias crônicas) estão diminuindo, o que sugere que o enfrentamento está ocorrendo na direção certa. Por consequência, o número de portadores de DCNT que requerem atendimento tende a aumentar. O congestionamento do SUS para consultas, exames especializados e cirurgias mostram a carga que essas doenças causam ao sistema público de saúde e indicam a necessidade de organizar, qualificar e ampliar o a prevenção sobre os fatores de risco e a redução da incidência dessas doenças. Isso fica evidente nos valores significativamente maiores no tratamento de infarto e AVC, enquanto para diabetes esses valores representam uma taxa menor. Sendo a diabetes fator de risco para inúmeras doenças, não apenas as doenças cardiovasculares, é necessário intervir antes das complicações, contribuindo para a redução dos custos na saúde.

\section{REFERÊNCIAS}

JANINI, Janaina Pinto; BESSLER, Danielle; VARGAS, Alessandra Barreto de. Educação em saúde e promoção da saúde: impacto na qualidade de vida do idoso. Saúde debate, Rio de Janeiro, v. 39, n. 105, p. 480-490, 2015.

SIQUEIRA, Alessandra de Sá Earp; SIQUEIRA-FILHO, Aristarco Gonçalves de; LAND, Marcelo Gerardin Poirot. Analysis of the Economic Impact of Cardiovascular Diseases in the Last Five Years in Brazil. Arq. Bras. Cardiol., São Paulo, v. 109, n. 1, p. 39-46, 2017.

RIBEIRO, Antonio Luiz P.; DUNCAN, Bruce B.; BRANT, Luisa C. C.; LOTUFO, Paulo A.; MILL, José Geraldo, BARRETO, Sandhi M. Cardiovascular Health in Brazil Trends and Perspectives. Circulation, v. 4, n. 133, p. 422-433, 2016.

LOTUFO, Paulo Andrade; GOULART, Alessandra Carvalho; PASSOS, Valéria Maria de Azeredo; SATAKE, Fabio Mitsuhiro; SOUZA, Maria de Fátima Marinho de; FRANÇA, Elizabeth Barbosa; RIBEIRO, Antônio Luiz Pinho; BENSENÕR, Isabela Judith Martins. Doença cerebrovascular no Brasil de 1990 a 2015: Global Burden of Disease 2015. Rev. bras. epidemiol., São Paulo, v. 20, supl. 1, p. 129-141, 2017.

SILOCCHI, Cassiane; JUNGES, José Roque. EQUIPES DE ATENÇÃO PRIMÁRIA: DIFICULDADES NO CUIDADO DE PESSOAS COM DOENÇAS CRÔNICAS NÃO TRANSMISSÍVEIS. Trab. educ. saúde, Rio de Janeiro, v. 15, n. 2, p. 599-615, 2017. 
MALTA, Deborah Carvalho; FRANÇA, Elisabeth; ABREU, Daisy Maria Xavier; PERILLO, Rosângela Durso; SALMEN, Maíra Coube; TEIXEIRA, Renato Azeredo; PASSOS, Valeria; SOUZA, Maria de Fátima Marinho; MOONEY, Meghan; NAGHAVI, Mohsen. Mortality due to noncommunicable diseases in Brazil, 1990 to 2015, according to estimates from the Global Burden of Disease study. São Paulo Med. J., São Paulo, v. 135, n. 3, p. 213-221, 2017.

MEDINA, Maria Guadalupe; AQUINO, Rosana; VILASBÔAS, Ana Luiza Queiroz; MOTA, Eduardo; PINTO JÚNIOR, Elzo Pereira; LUZ, Leandro Alves da; ANJOS, Davllyn Santos Oliveira dos; PINTO, Isabela Cardoso de Matos. Promoção da saúde e prevenção de doenças crônicas: o que fazem as equipes de Saúde da Família?. Saúde debate, Rio de Janeiro, v. 38, n. spe, p. 69-82, 2014.

MALTA, Deborah Carvalho; STOPA, Sheila Rizzato; SZWARCWALD, Celia Landmann; GOMES, Nayara Lopes; SILVA JÚNIOR, Jarbas Barbosa; REIS, Ademar Arthur Chioro dos. A vigilância e o monitoramento das principais doenças crônicas não transmissíveis no Brasil - Pesquisa Nacional de Saúde, 2013. Rev. bras. epidemiol., São Paulo, v. 18, supl. 2, p. 3-16, 2015.

MINISTÉRIO DA SAÚDE. Secretaria de Vigilância em Saúde. Departamento de Análise de Situação de Saúde. Plano de ações estratégicas para o enfrentamento das doenças crônicas não transmissíveis (DCNT) no Brasil 2011-2022. Brasília: Ministério da Saúde, 2011.

ORGANIZAÇÃO MUNDIAL DE SAÚDE. Noncommunicable diseases country profiles 2014.210 p., 2014.

TANAKA, Oswaldo Yoshimi. Avaliação da atenção básica em saúde: uma nova proposta. Saude soc., São Paulo, v. 20, n. 4, p. 927-934, 2011.

RADOVANOVIC, Cremilde Aparecida Trindade; SANTOS, Lucimary Afonso dos; CARVALHO, Maria Dalva de Barros; MARCON, Sonia Silva. Arterial Hypertension and other risk factors associated with cardiovascular diseases among adults. Rev. Latino-Am. Enfermagem. Ribeirão Preto, v. 22, n. 4, p. 547-553, 2014.

MATAVELLI, lara Silva; JUDICE, Eduardo Luís Del; MATAVELLI, Rafael; HUNGER, Marcelo Studart; MARTELLI, Anderson. The Regular Practice of Physical Exercise to Control Systemic Arterial Hypertension: A Literature Review. Revista Brasileira de Ciências da Saúde. Mogi Guaçu, v. 18, n. 4, p. 359-366, 2014.

SHAW, J.E.; SICREE, R.A.; ZIMMET, P.Z.. Global estimates of the prevalence of diabetes for 2010 and 2030. Diabetes Research and Clinical Practice., v. 87, n. 1, p. 4-14, 2010, https://doi.org/10.1016/i.diabres.2009.10.007. 